ORIGINAL RESEARCH

\title{
Use of the Physician Orders for Life-Sustaining Treatment among California Nursing Home Residents
}

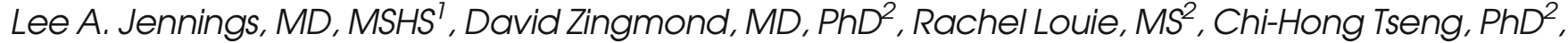 \\ Judy Thomas, $\mathrm{JD}^{3}$, Kate O'Malley, RN, MS', and Neil S. Wenger, MD, MPH²
}

'David Geffen School of Medicine at UCLA, Division of Geriatrics, Los Angeles, CA, USA; ${ }^{2}$ David Geffen School of Medicine at UCLA, Division of General Internal Medicine and Health Services Research, Los Angeles, CA, USA; ${ }^{3}$ Coalition for Compassionate Care of California, Sacramento, CA, USA; ${ }^{4}$ California HealthCare Foundation, Oakland, CA, USA.

BACKGROUND: Physician Orders for Life-Sustaining Treatment (POLST) is a tool that facilitates the elicitation and continuity of life-sustaining care preferences. POLST was implemented in California in 2009, but how well it disseminated across a large, racially diverse population is not known and has implications for end-of-life care.

OBJECTIVE: To evaluate the use of POLST among California nursing home residents, including variation by resident characteristics and by nursing home facility.

DESIGN: Observational study using California Minimum Data Set Section S.

PARTICIPANTS: A total of 296,276 people with a stay in 1,220 California nursing homes in 2011 .

MAIN MEASURES: The proportion of residents with a completed POLST (containing a resuscitation status order and resident/proxy and physician signatures) and relationship to resident characteristics; change in POLST use during 2011; and POLST completion and unsigned forms within nursing homes.

KEY RESULTS: During 2011, POLST completion increased from 33 to $49 \%$ of California nursing home residents. Adjusting for age and gender using a mixed-effects logistic model, long-stay residents were more likely than short-stay residents to have a completed POLST $[\mathrm{OR}=2.36$ (95 \% CI 2.30, 2.42)]; severely cognitively impaired residents were less likely than unimpaired to have a completed POLST [OR = 0.89 (95 \% CI 0.87, 0.92)]; and there was little difference by functional status. There was no difference in POLST completion among White non-Hispanic, Black, and Hispanic residents. Variation in POLST completion among nursing homes far exceeded that attributable to resident characteristics with $40 \%$ of facilities having $\geq 80 \%$ of long-stay residents with a completed POLST, while $20 \%$ of facilities had $\leq 10 \%$ of long-stay residents with a completed POLST. Thirteen percent of nursing home residents had a POLST containing a resuscitation preference but lacked a signature, rendering the POLST invalid.

CONCLUSIONS: Statewide nursing home data show broad uptake of POLST in California without racial

Electronic supplementary material The online version of this article (doi:10.1007/s11606-016-3728-9) contains supplementary material, which is available to authorized users.

Received September 18, 2015

Revised March 9, 2016

Accepted April 22, 2016

Published online May 17, 2016 disparity. However, variation in POLST completion among nursing homes identifies potential areas for quality improvement.

KEY WORDS: end-of-life care; nursing home; long-term care; quality improvement.

J Gen Intern Med 31(10):1119-26

DOI: $10.1007 / \mathrm{s} 11606-016-3728-9$

๑) Society of General Internal Medicine 2016

\section{INTRODUCTION}

Physician Orders for Life-Sustaining Treatment (POLST) facilitates elicitation of life-sustaining treatment preferences from patients and is designed to improve the transition of these preferences, in the form of physician orders, across care settings. POLST captures preferences concerning cardiopulmonary resuscitation, overall aggressiveness of care, hospital transfer, and artificial nutrition. ${ }^{1}$ The document is particularly useful for residents of nursing facilities because many residents live in compromised health states and would not desire burdensome treatments ${ }^{2}$ and also because it is common for nursing home residents to transition to the emergency room and the hospital ${ }^{3}$ where resuscitation and end of life treatments can be guided by the POLST.

In 2009, POLST became a legal clinical tool in California, and its use was propelled by a grassroots community coalition intervention. ${ }^{4}$ In 2011, California became the only state thus far to collect information about POLST in the Long-Term Care Minimum Data Set (MDS), the nationwide survey that collects information on all nursing home residents. This affords the opportunity to understand the level of POLST uptake within a statewide nursing home population during the third year of implementation in California and to evaluate the uniformity of POLST use across nursing homes and the relationship of POLST use to resident characteristics. Furthermore, the MDS survey permits exploration of POLST implementation for quality improvement, for example, how often documents lacked resident/proxy or physician signatures and whether deficiencies were concentrated in certain nursing homes.

We used the $2011 \mathrm{MDS}$ to evaluate the use of POLST among nursing home residents in California in 2011, variation 
in use at the level of the nursing home, and the relationship of POLST use with resident characteristics.

\section{METHODS}

We evaluated the use of POLST in all 296,276 residents who had at least one stay in a California nursing home in 2011, defined as the completion of at least one MDS assessment in 2011.

The MDS is a standardized, federally mandated screening and health status assessment tool completed for all residents in a Medicare and/or Medicaid-certified long-term care facility. ${ }^{5}$ The MDS collects detailed demographic and clinical information on admission to the nursing home, quarterly, if there is a significant change in clinical status and at discharge or death. A section containing questions about the use of POLST (Section S) was added to the California MDS on October 1, 2010. Questions in California MDS Section S include whether or not the resident completed a POLST; the content of the POLST form including resuscitation status (do not resuscitate vs. attempt resuscitation in POLST Section A), desired level of medical intervention (comfort measures vs. limited interventions vs. full treatment in POLST Section B), and choice about artificial nutrition, including feeding tubes (no artificial nutrition vs. trial of artificial nutrition vs. long-term artificial nutrition in POLST Section C). California MDS Section S also collected additional information contained on the POLST form about whether or not the resident had an advance directive, with whom the POLST was discussed, and whether the POLST form was signed by the resident (or a legally recognized decision-maker) and physician (POLST Section D). The advance directive item was added to the California POLST when the form was revised on April 1, 2011,7 (Online Appendix Figure 1 and Online Appendix Table 1).

Section S of the MDS was linked to other data in the MDS to understand the length of nursing home stay, number of nursing home admissions in 2011, demographic characteristics (age, gender, and race or ethnic group), functional status, and cognition. Cognitive status was measured using the validated MDS 3.0 Brief Interview for Mental Status (BIMS), ${ }^{8}$ which was present for $69 \%$ of residents, supplemented when missing with the staff assessment for $26 \%$ or an indication that the resident was comatose $(0.4 \%)$. Using this combined variable, cognitive impairment was defined as none (BIMS score $13-15$ or not impaired by staff assessment), mild to moderate (BIMS score $8-12$ ), or severe (BIMS score $0-7$ or impaired by staff assessment or comatose). To define functional status, we used the validated MDS Activities of Daily Living (ADL) scale, which ranges from 0 (independent in all seven ADLs) to 28 (total dependence in all seven ADLs). ${ }^{9}$ Demographic and clinical variables and Section $S$ variables were drawn from the same MDS assessment except if ADL and cognitive assessments were unavailable in which case they were taken from another MDS assessment collected during a 90-day window around the Section S assessment. For $4.9 \%$ of residents with completed Section S, ADL or cognitive assessment was unavailable.

We used the CMS definition for long-stay and short-stay nursing home residents, which defines long stay as residing in the nursing home more than 100 consecutive days. Where necessary, we also used 2010 MDS data to define the length of stay. ${ }^{10}$ Length of stay was defined using the nursing home admission associated with the last MDS Section S completed in 2011 .

\section{Analyses}

We evaluated whether Section S was completed in any MDS assessment ${ }^{11}$ (see Online Appendix Table 2) and, for those with a MDS Section S, whether the resident had a completed POLST, defined as having an order in POLST Section A and signatures of both the resident (or appropriate proxy) and physician. ${ }^{12}$ Using this definition of a completed POLST, we used chi-square tests to compare POLST completion by whether the MDS Section S was the first (median date April 2,2011 ) or the last MDS Section S (median date October 4, 2011) completed for the resident during the 2011 year. Median number of MDS Section S completions was 3 (IQR 2, 5); $89 \%$ of residents had more than one Section S completed in 2011.

When MDS Section S data indicated that a POLST was present but POLST Section A had no content or the physician or resident/proxy signature was missing, the POLST form was considered to be incomplete. When MDS Section S described a POLST that contained POLST Section A content (resuscitation status) but was missing either resident/proxy or physician signature(s), we classified the document as incomplete because of a missing signature (an unsigned POLST). We also described with whom the POLST form was discussed and responses to the advance directive item contained in POLST Section D. These Section D items are described only for residents with a completed POLST. ${ }^{13}$

After examining whether residents had a completed POLST, with whom the POLST was discussed, and whether an advance directive was reviewed, we stratified these data by length of stay and examined demographic and clinical characteristics between nursing home residents who completed a POLST and those who did not.

We investigated the use of POLST at the nursing home level by clustering residents within nursing home and then examining the proportion of residents within a nursing home who completed a POLST, overall and stratified by long-stay and short-stay residents. This was computed for the first MDS Section S completed in 2011 and last MDS Section S completed in 2011. We also examined the proportion of residents within a nursing home with an unsigned POLST.

Lastly, we used a mixed effects multivariate logistic regression model to predict completion of a POLST including resident age, gender, race/ethnicity, length of nursing home stay, cognitive status, and ADL score as fixed effects. The model 
excluded the 14,148 residents with missing cognitive or functional status so we repeated the model with the full cohort adding a category for missing data for each variable and found similar results. The few residents $(N=7622,2.6 \%)$ with missing race/ethnicity were grouped with "other" race/ethnicity for analyses. We also included nursing home facility as a random effect in the model. We computed McFadden's $\mathrm{R}^{2}$ for the model with and without the nursing home random effect to derive variation in POLST completion explained by resident characteristics alone versus variation in POLST completion explained by the addition of nursing home facility to the model. Lastly, we repeated the mixed-effect model on the cohort stratified by long and short length of stay.

All analyses were performed using STATA IC version $13 .{ }^{14}$ This project was approved by the UCLA Institutional Review Board.

\section{RESULTS}

Of the 296,276 people with a California nursing home stay in 2011, for $98 \%$ some part of MDS Section S was completed. The 289,753 residents in 1220 nursing facilities with Section $\mathrm{S}$ data had a mean age of 78 years, $61 \%$ were female, $67 \%$ were white non-Hispanic, and two-thirds were short-stay. Thirty percent of residents had extensive or total dependence in all ADLs and one-third were severely cognitively impaired. In bivariate analyses, demographic and clinical characteristics were similar between those with a completed POLST (resuscitation status completed and POLST signed by resident/proxy and physician) and those with an incomplete
POLST (no resusCitation status or unsigned) or no POLST, although there were statistically significant small differences (Table 1).

\section{POLST Completion during 2011}

Over the 1-year period of 2011, POLST completion increased. In the first MDS Section S completed in 2011, $33 \%$ of residents $(N=95,168)$ had a completed POLST, whereas $49 \%$ of residents $(N=142,672)$ had a completed POLST in the last Section S (Fig. 1). POLST completion was greater among long-stay residents than short-stay residents in both the first and last MDS Section S (First Section S, long stay $44 \%$ vs. short stay $29 \%, p<0.001$; Last Section S, 57 vs. $45 \%$, respectively, $p<0.001)$. POLST completion was lowest (33 \%, Last Section $\mathrm{S}$ ) among those residents with very short length of stay (1-7 days) (Online Appendix Table 4). Five percent of residents $(N=13,753$, last Section $\mathrm{S})$ completed POLST Section A (resuscitation status) but did not complete either POLST Section B (level of intervention) or POLST Section C (artificial nutrition).

\section{POLST Completion at the Nursing Home Level}

POLST completion was heterogeneous across nursing homes in both the first and last MDS Section S completed in 2011 (Fig. 2). In the last Section S data, $93 \%$ of facilities had at least one resident with a completed POLST, yet one in five facilities $(N=265)$ had fewer than $10 \%$ of residents with a completed POLST (excludes 16 facilities with fewer than 20 residents, $N=1204$ facilities). Nursing homes had more completed POLSTs among long-stay residents. In the last Section S,

Table 1 Resident characteristics by POLST completion (Last Section S in 2011)*

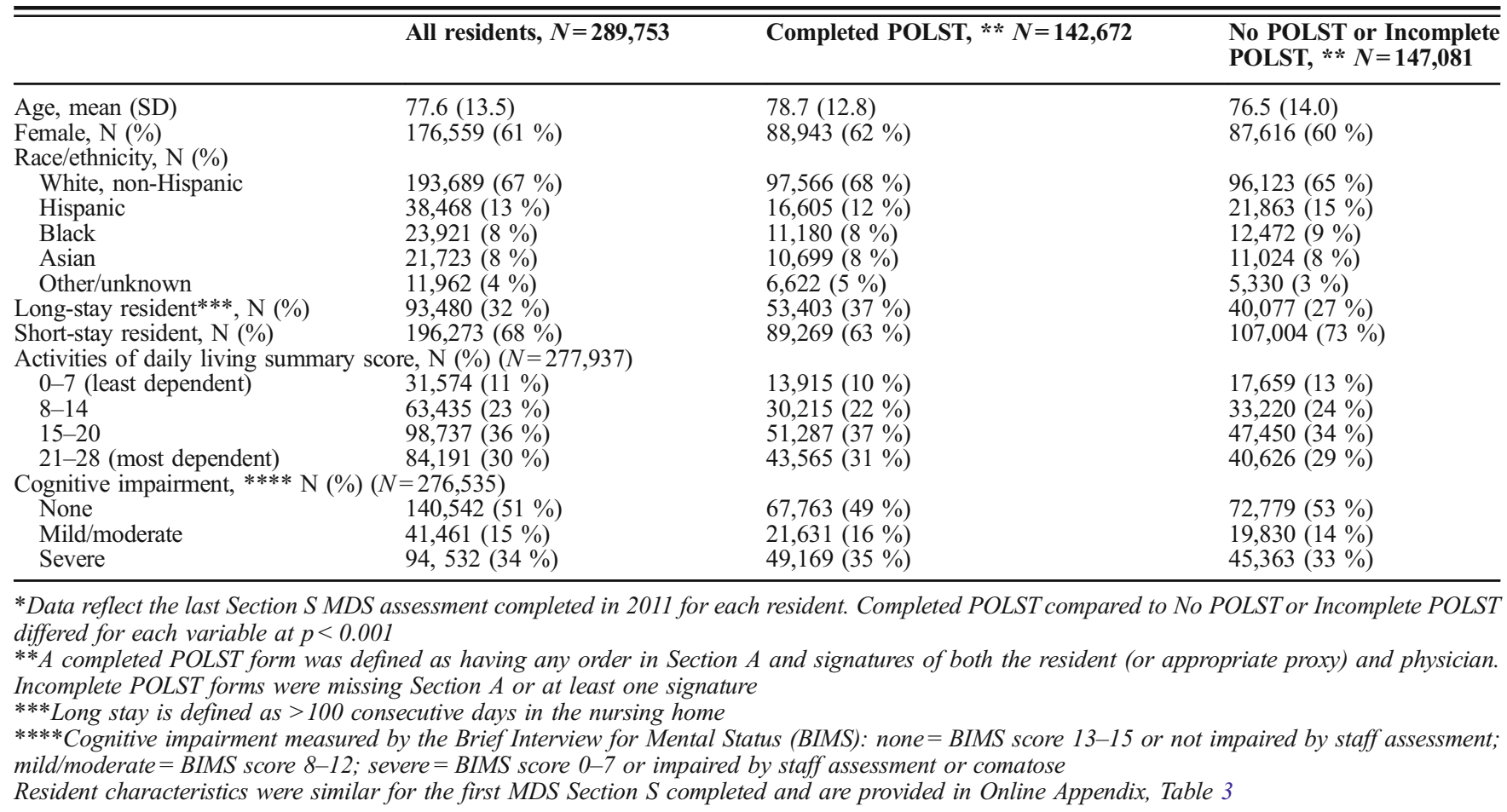



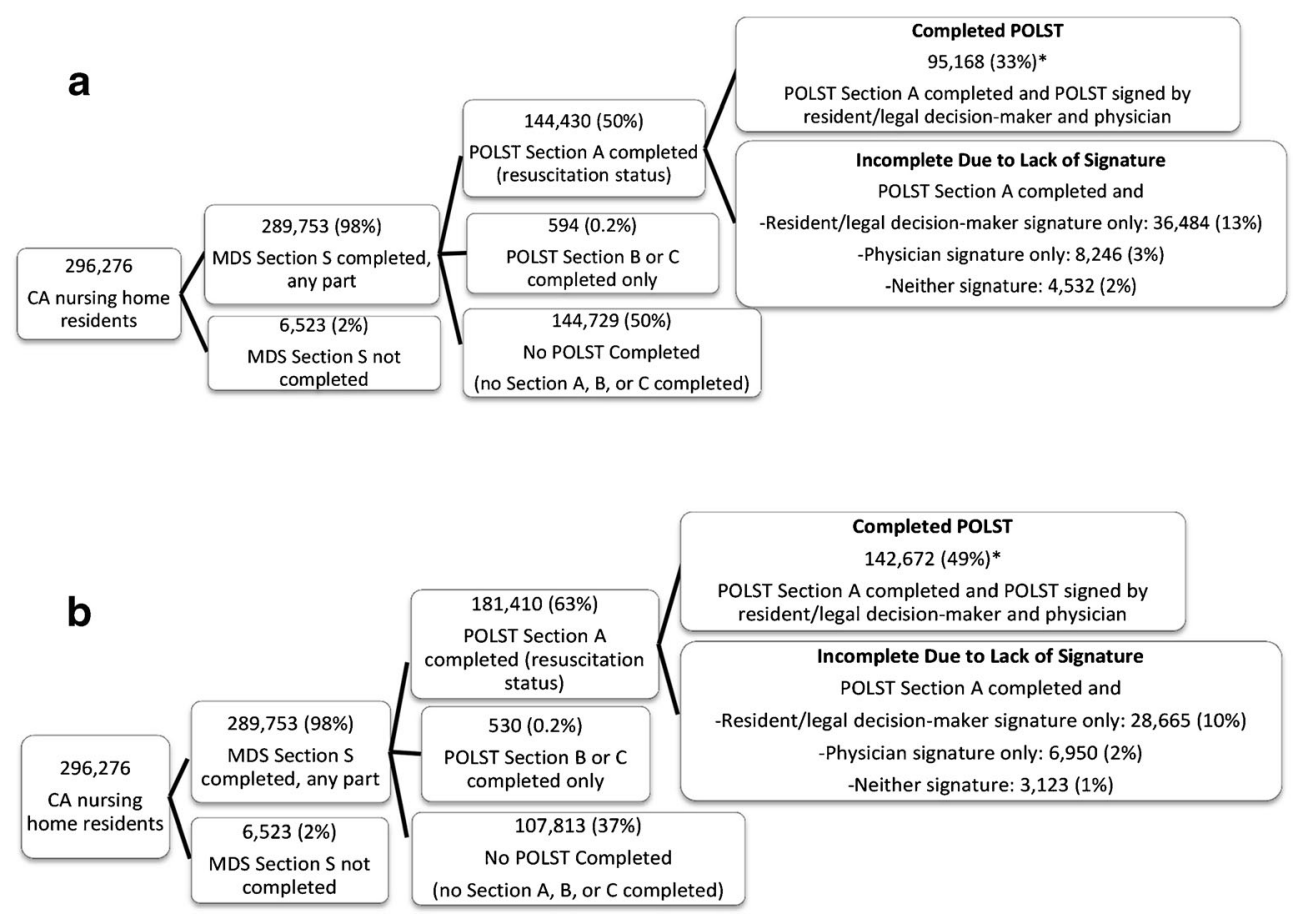

Fig. 1 POLST use in California nursing homes in 2011. (a) First MDS Section S completed in 2011: POLST Sections A, B, and C and signatures. (b) Last MDS Section S completed in 2011: POLST Sections A, B, and C and signatures Completed POLSTs are defined as those with Section A (resuscitation status) completed and signed by the resident/proxy and physician. POLST Section A contains preferences regarding resuscitation status, POLST Section B contains preferences regarding level of intervention, and POLST Section C contains preferences regarding artificial nutrition (2011 California POLST form provided in Online Appendix Figure 1). Denominator for all percentages is $N=289,753$ (beyond the first branch in the tree). *Compared using the chi-square test, $p<0.0001$

$40 \%$ of facilities $(N=445)$ had $\geq 80 \%$ of long-stay residents with a completed POLST, while $17 \%$ of facilities $(N=179)$ had $\geq 80 \%$ of short-stay residents with a completed POLST. However, $20 \%$ of facilities $(N=221)$ had $\leq 10 \%$ of long-stay residents with a completed POLST (Fig. 2).

\section{Unsigned POLST Forms}

The percentage of unsigned POLSTs (resuscitation status and no resident/proxy or physician signature) decreased during 2011 from $34 \%$ (first Section S) to $21 \%$ (last Section S) (Fig. 1). The majority of unsigned POLSTs were missing physician signatures $(N=31,788$, last Section $\mathrm{S})$, and most forms missing physician signatures were completed by shortstay residents $(N=25,927,82 \%)$ with lengths of stay less than 2 weeks $(N=15,589,49 \%)$. In 207 facilities (17\%), $\geq 20 \%$ of residents had a POLST form missing a physician signature (last Section S, Fig. 3), and 6 facilities had at least half of residents with forms missing a physician signature. Few POLST forms without care preferences (no POLST Section A, B, or C completed) were signed $(1 \%, N=2,116$ last Section $\mathrm{S}$ ).

\section{Discussion about POLST and Advance Directives}

Among residents with a completed POLST, the MDS recorded that the POLST was discussed with the resident in $45 \%$ of cases and with the legal decision-maker in $54 \%$ (Last
Section S). POLST was more often discussed with the legal decision-maker for long-stay residents compared with shortstay residents (70 vs. $44 \%, p<0.001$ ).

The advance directive question was available for 126,462 residents with a completed POLST on the last MDS Section S because this item was not present on the 2009 POLST form. Among these residents, Section $\mathrm{S}$ recorded that an advance directive was available and reviewed for $26 \%$ of residents, an advance directive was not available for $7 \%$, and there was no advance directive for $43 \%$; this section was not completed for $24 \%$ of residents. Long-stay residents more often had an advance directive that was reviewed (33 vs. $21 \%, p<0.001$ ) (Online Appendix Table 5).

\section{Factors Associated with Completing a POLST}

In the mixed-effects multivariate logistic regression model, long length of stay was independently associated with completion of a POLST [odds ratio (OR) 2.36, $95 \%$ confidence interval (CI) $2.30,2.42]$. Older age and female gender were also associated with POLST completion, although ORs approached one. Residents with severe cognitive impairment were less likely to have a completed POLST compared to those without cognitive impairment after adjusting for other covariates (OR 0.89, $95 \%$ CI $0.87,0.92)$. This relationship was more profound for long-stay residents (OR 0.76, $95 \%$ CI 0.72, 0.80 vs. OR $0.95,95 \%$ CI $0.92,0.98)$ in the model stratified by 
a

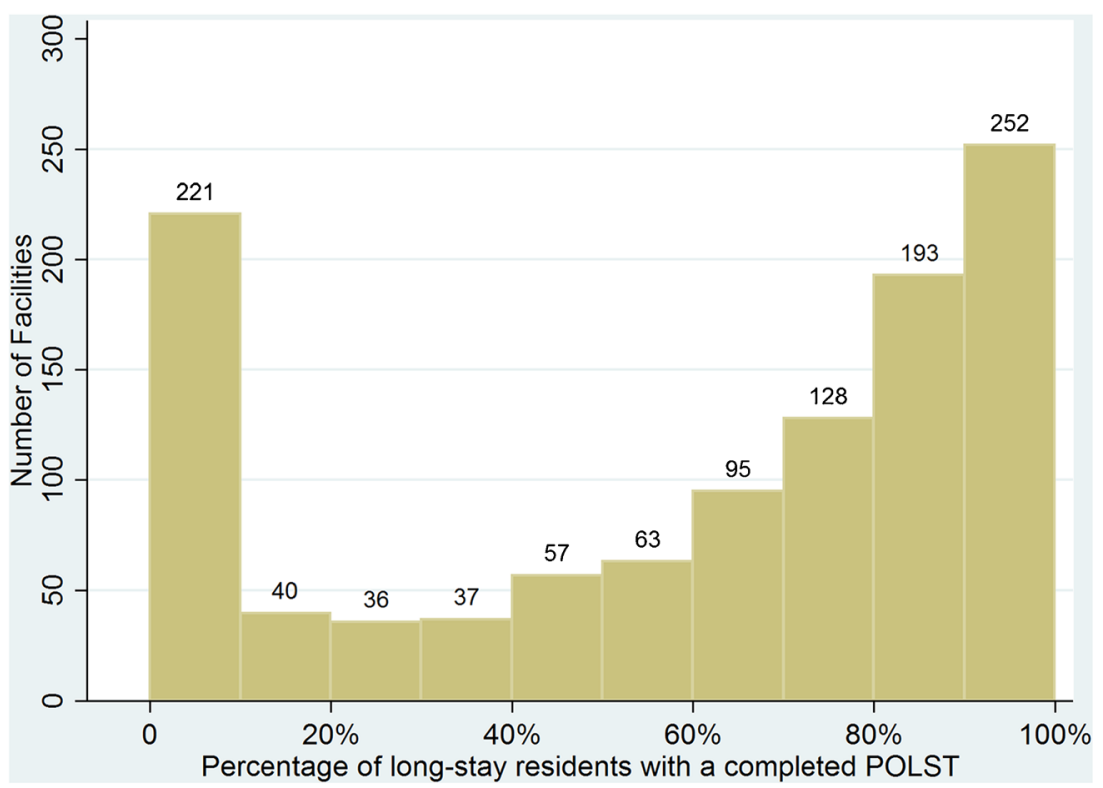

b

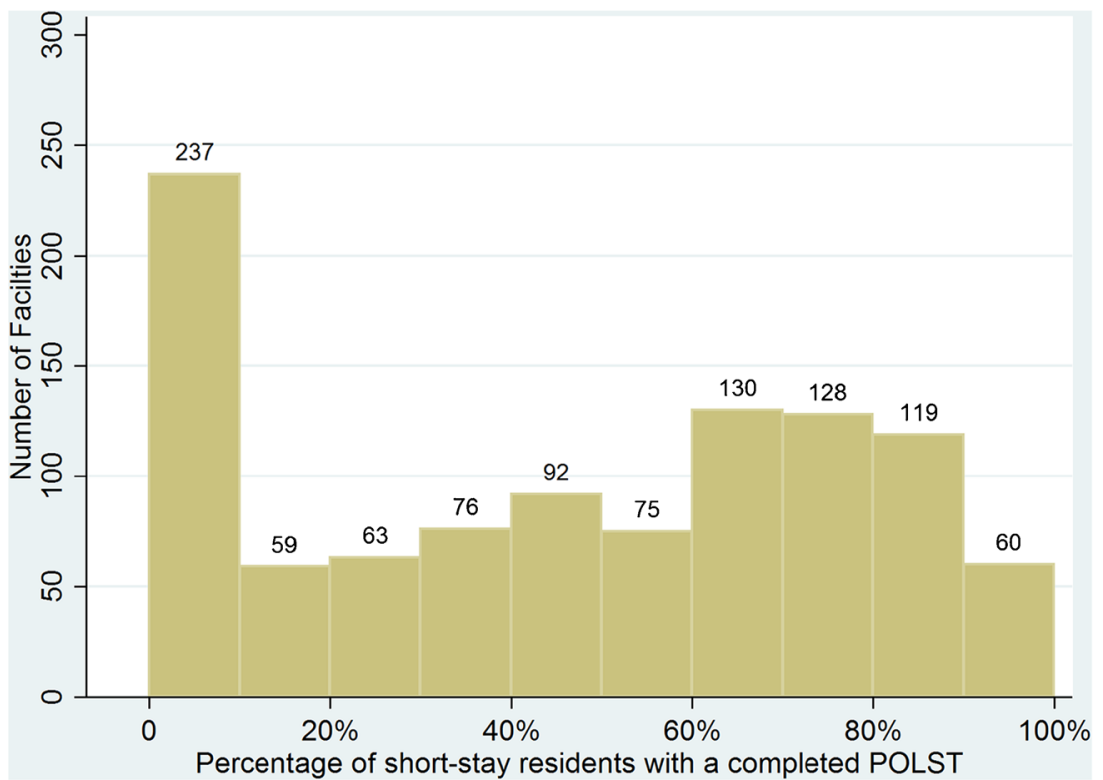

Fig. 2 POLST completion by nursing home facility. Legend 2a: $N=1,122$ facilities. Excludes 98 facilities with fewer than 20 long-stay residents. Legend 2b: $N=1,039$ facilities. Excludes 181 facilities with fewer than 20 short-stay residents. Data from the last MDS Section $S$ completed in 2011 for each resident

length of stay. Functional impairment was not independently related to POLST completion in a consistent way. Hispanic and Black race/ethnicity was not different from White in predicting whether a resident completed a POLST, although Asians and other race/ethnicity were more likely to have a POLST. (Table 2). McFadden's $\mathrm{R}^{2}$ for the resident characteristic model was 0.016; after the nursing home random effect was added to the model, McFadden's $\mathrm{R}^{2}$ was 0.36 , indicating that nearly all the variation in POLST completion explained by the model was at the level of the nursing home.

\section{DISCUSSION}

This analysis of the first population-based, statewide data of POLST completion in nursing homes demonstrates widespread uptake of this end-of-life transition intervention in California during the third year after implementation. Last available MDS Section S data in 2011 show that $49 \%$ of nursing home residents in California completed a POLST, and $93 \%$ of California nursing homes had at least one resident with a POLST. Furthermore, the quality of POLST completion, according to these MDS data, appears to be high, with last available 2011 data showing that only $13 \%$ of residents 


\section{Incomplete POLST Forms by Nursing Home}

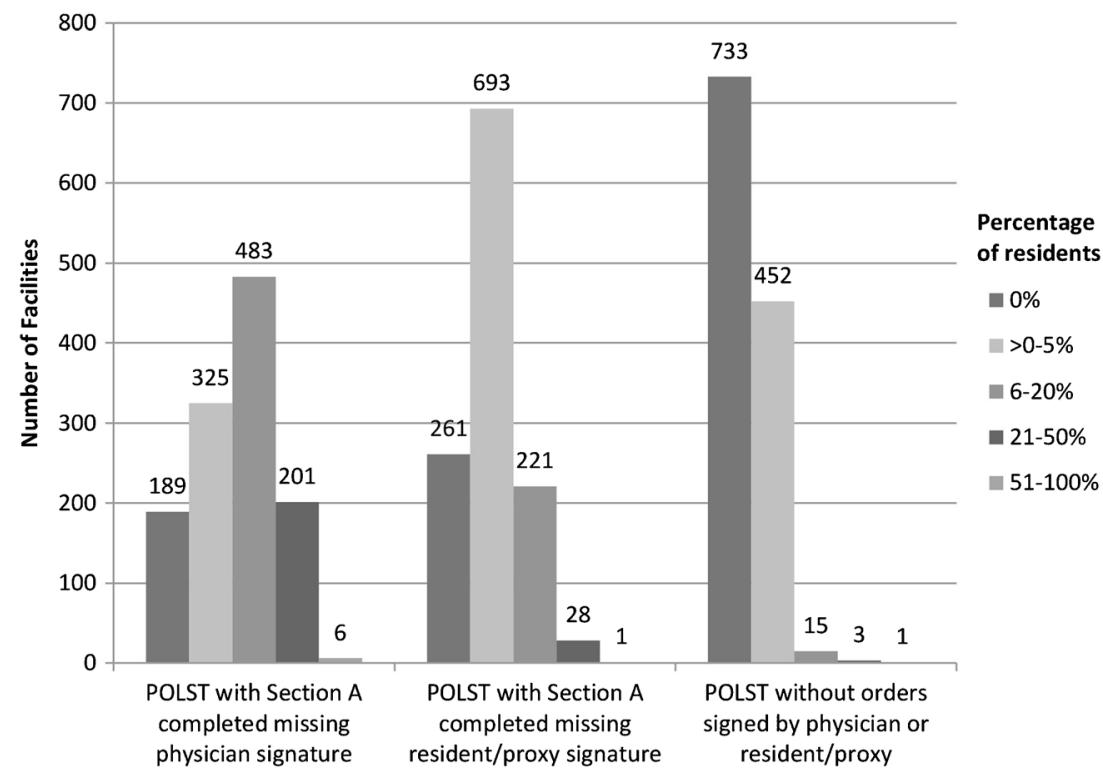

Fig. 3 Incomplete POLST forms by nursing home Figure shows the number of nursing homes $(N=1204)$ with no residents, $<5$, 6-20, 2150 , and 51-100\% of residents with POLST forms with Section A completed but missing a physician or resident/proxy signature or POLST forms signed by a physician or resident/proxy that contain no orders. Excludes 16 facilities with fewer than 20 residents. Data are from the last MDS Section S completed in 2011 for each resident

had a POLST including a resuscitation order with a missing signature; only $1 \%$ had a POLST with a signature without order information; and only $5 \%$ had a POLST that lacked information about the level of the intervention or artificial nutrition. It is particularly noteworthy that POLST uptake varied by clinically appropriate factors such as nursing home length of stay and age, but not by race/ethnicity, which suggests successful implementation across diverse communities. This finding stands in stark contrast to a plethora of prior data showing disparity in advance care planning and end-of-life care across ethnicities. ${ }^{15-17}$ These findings show that a broad, rather complex public health intervention can be disseminated widely and effectively in a relatively brief period of time across a large, racially diverse state.

The California POLST dissemination was a broad-based statewide effort that included a coordinated effort among stakeholders throughout the state, a coordinating organization supported with funding from a grant-making organization focused on healthcare, combined with a grassroots initiative. California state law (Assembly Bill 3000) in 2008 authorized the use of POLST throughout the state, effective January 1, 2009. ${ }^{12}$ The Coalition for Compassionate Care of California (CCCC), supported by the California HealthCare Foundation, established a statewide POLST task force and developed tools and resources for POLST implementation. Eighteen community coalitions used educational and teaching materials for patients, clinicians, and facilities to teach the appropriate use of POLST and integrate POLST into clinical care. While it is not possible to directly link any particular step of the California dissemination effort with the broad uptake of POLST in the state, these data suggest that this model was successful.
However, the paradoxical relationship of POLST completion to cognition, especially among long-stay residents, merits further exploration. While this analysis did not explore the orders contained within the POLST, most POLST forms from nursing home residents contain orders to avoid resuscitation. ${ }^{18,19}$ Surveys of older people ${ }^{20}$ and nursing home residents ${ }^{21}$ consistently show that people with greater functional disability tend to desire less aggressive care, and studies of older people looking ahead to future compromised health states demonstrate elicited preferences focusing on comfortoriented care and non-aggressive treatments. ${ }^{22,23}$ Possible explanations for fewer POLSTs among compromised residents include the unavailability of proxy decision-makers, family members' uncertainty about loved ones' wishes, or perhaps that long-stay severely compromised nursing home residents previously indicated a preference to live in this health state. Exploration is needed to further understand why longstay nursing home residents with greater cognitive impairment have less POLST use to determine if an advance care planning intervention is needed to better meet the needs of this vulnerable group.

The multivariate logistic regression model demonstrates that nursing home, rather than resident characteristics, largely predicts POLST completion, and MDS data show heterogeneous uptake of POLST across nursing homes. Variable uptake of end-of-life interventions has been previously demonstrated and is an aspect of long-term care in need of improvement. ${ }^{20}$ These data suggest areas for possible quality improvement, such as facilities that are slow adopters of POLST and unsigned forms. An unsigned POLST in the patient record may reflect a POLST form that is in process, especially for 
Table 2 Logistic regression model for completion of a POLST for the full population and stratified by long and short stay

\begin{tabular}{|c|c|c|c|}
\hline & \multicolumn{3}{|c|}{ Completion of a POLST OR (95 \% CI) } \\
\hline & $\begin{array}{l}\text { Overall, } \\
N=275,605\end{array}$ & $\begin{array}{l}\text { Long-stay, } \\
N=92,376\end{array}$ & $\begin{array}{l}\text { Short-stay, } \\
N=183,229\end{array}$ \\
\hline $\begin{array}{l}\text { Long-stay } \\
\text { resident }\end{array}$ & $\begin{array}{l}2.36(2.30 \\
2.42)\end{array}$ & - & - \\
\hline \multicolumn{4}{|c|}{ Cognitive impairment } \\
\hline None & REF & REF & REF \\
\hline Mild/moderate & $\begin{array}{l}0.98(0.95 \\
1.01)\end{array}$ & $\begin{array}{l}0.88(0.83 \\
0.93)\end{array}$ & $\begin{array}{l}1.01(0.98, \\
1.05)\end{array}$ \\
\hline Severe & $\begin{array}{l}0.89(0.87 \\
0.92)\end{array}$ & $\begin{array}{l}0.76(0.72 \\
0.80)\end{array}$ & $\begin{array}{l}0.95(0.92 \\
0.98)\end{array}$ \\
\hline \multicolumn{4}{|l|}{ ADL score } \\
\hline $\begin{array}{l}0-7 \text { (least } \\
\text { dependent) }\end{array}$ & REF & REF & REF \\
\hline $8-14$ & $\begin{array}{l}1.04(1.00, \\
1.08)\end{array}$ & $\begin{array}{l}1.14(1.06, \\
1.24)\end{array}$ & $\begin{array}{l}1.01(0.97, \\
1.06)\end{array}$ \\
\hline $15-20$ & $\begin{array}{l}1.03(0.99 \\
1.07)\end{array}$ & $\begin{array}{l}1.19(1.10, \\
1.27)\end{array}$ & $\begin{array}{l}0.97(0.93 \\
1.02)\end{array}$ \\
\hline $\begin{array}{l}21-28 \text { (most } \\
\text { dependent) }\end{array}$ & $\begin{array}{l}0.97(0.93 \\
1.01)\end{array}$ & $\begin{array}{l}1.07(0.99 \\
1.15)\end{array}$ & $\begin{array}{l}0.94(0.83 \\
0.996)\end{array}$ \\
\hline $\begin{array}{l}\text { Age (per } \\
\text { additional } 5 \\
\text { years) }\end{array}$ & $\begin{array}{l}1.03(1.03, \\
1.04)\end{array}$ & $\begin{array}{l}1.04(1.04, \\
1.05)\end{array}$ & $\begin{array}{l}1.03(1.03, \\
1.04)\end{array}$ \\
\hline Female & $\begin{array}{l}1.02(1.003, \\
1.05)\end{array}$ & $\begin{array}{l}1.01(0.98, \\
1.05)\end{array}$ & $\begin{array}{l}1.03(1.01, \\
1.06)\end{array}$ \\
\hline \multicolumn{4}{|l|}{ Race/ethnicity } \\
\hline White, non- & REF & REF & REF \\
\hline \multicolumn{4}{|l|}{ Hispanic } \\
\hline Hispanic & $\begin{array}{l}0.99(0.96, \\
1.02)\end{array}$ & $\begin{array}{l}0.98(0.93 \\
1.04)\end{array}$ & $\begin{array}{l}0.995(0.96, \\
1.04)\end{array}$ \\
\hline Black & $\begin{array}{l}1.03(0.99 \\
1.07)\end{array}$ & $\begin{array}{l}1.01(0.95 \\
1.09)\end{array}$ & $\begin{array}{l}1.04(0.99 \\
1.10)\end{array}$ \\
\hline Asian & $\begin{array}{l}1.05(1.01, \\
1.10)\end{array}$ & $\begin{array}{l}1.05(0.97 \\
1.04)\end{array}$ & $\begin{array}{l}1.05(0.99 \\
1.10)\end{array}$ \\
\hline Other/unknown & $\begin{array}{l}1.07(1.02 \\
1.13)\end{array}$ & $\begin{array}{l}1.06(0.96 \\
1.18)\end{array}$ & $\begin{array}{l}1.09(1.02, \\
1.16)\end{array}$ \\
\hline
\end{tabular}

The logistic regression model includes the variables listed plus the nursing home facility as a random effect. McFadden's pseudo $R^{2}$ for the models range from 0.34 to 0.38 (likelihood ratio test of the nursing home random effect $p<0.001$ )

Odds ratios $(O R s)$ in bold are significantly different from the reference at $p<0.05$. $C I=$ confidence interval

Model of completion of a POLST excludes $4.9 \%(N=14,148)$ of residents missing cognitive status or ADL score and excludes one nursing home facility ( $N=1,219$ nursing facilities). Long-stay model excludes 1104 residents (1.2\%) and short-stay model excludes 13,044 residents $(6.6 \%)$

Long stay is defined as $>100$ consecutive days in the nursing home. Data are from the last MDS Section S completed in 2011 for each resident

residents whose length of stay in the nursing home is very short and thus may be discharged prior to fully completing the form; however, because an unsigned POLST is not legally valid, it should not be stored in the patient record. The lack of nursing home characteristics within in the MDS data limits further exploration of facility-level characteristics that may be associated with slow adoption of the POLST.

Up-to-date data are needed to guide intervention, but these results demonstrate how MDS data can be used to target quality improvement. Exploring how agencies regulating long-term care in California can support high-quality POLST form completion may be helpful. Additionally, a registry of POLST forms containing a quality assessment component could help ensure forms contain complete, consistent information and appropriate signatures.
Section S data are collected within the context of a federally mandated survey with the advantage of covering the full population of nursing home residents in the state of California. The MDS 3.0, into which the POLST Section $\mathrm{S}$ items were embedded, has item-level kappa scores in the very good to excellent range, ${ }^{24}$ indicating good reliability of the covariates used in these analyses. In addition, the cognitive status and function measures have been demonstrated to be valid. ${ }^{24}$ The POLST items have not been tested for reliability, yet the high degree of internal consistency among the sections of the POLST form suggests reliable recording of these data. Furthermore, these findings are consistent with data obtained by a survey of a small sample of California nursing homes collected during the same time period. ${ }^{4}$ These data also have limitations. Since the POLST form can be completed prior to nursing home admission in another care setting, and MDS Section S does not record the date of POLST completion, it is not possible to know when a POLST form was completed with a resident. However, at the time these data were recorded, the POLST form had been available for clinical use in California for only 2 years. The greatest limitation of these data is that they cannot capture whether the completed POLST forms reflect highquality advance care planning conversations.

In conclusion, statewide nursing home data show wide and appropriate use of POLST in California in response to a multipronged dissemination intervention. During the third year after implementation of POLST, use of the tool was still rapidly increasing, although a significant minority of nursing homes was slow to adopt POLST. MDS data demonstrate targets for quality improvement that could be used to improve end-of-life care at particular nursing homes.

\section{Acknowledgments:}

We thank Debby Rogers, Tina Kruthoff, and James Booth of the Center for Health Care Quality, California Department of Public Health, for their assistance with data acquisition.

We presented this work as an oral presentation at the Society of General Internal Medicine Annual Meeting in Toronto, Canada, on April 23, 2015.

Support: The project was funded by the California HealthCare Foundation. Dr. Jennings was supported by the John A. Hartford Foundation as a UCLA Center of Excellence Scholar and by the UCLA Claude Pepper Older Americans Independence Center funded by the National Institute on Aging (5P30AG028748) and the NIH/National Center for Advancing Translational Science (NCATS) UCLA CTSI (UL1TROOO124).

Corresponding Author: Lee A. Jennings, MD, MSHS; David Geffen School of Medicine at UCLA, Division of Geriatrics, 10945 Le Conte Ave., Suite 2339, Los Angeles, CA 90095-1687, USA (e-mail: lajennings@mednet.ucla.edu).

Compliance with Ethical Standards:

Conflict of Interest: All of the authors declare that they have no conflicts of interest with regard to this manuscript. 


\section{REFERENCES}

1. Citko J, Moss AH, Carley M, Tolle S. The National Polst Paradigm Initiative, 2nd Edition \#178. Journal of Palliative Medicine. 2011;14(2):241-242.

2. Hickman SE, Nelson CA, Perrin NA, Moss AH, Hammes BJ, Tolle SW. A comparison of methods to communicate treatment preferences in nursing facilities: traditional practices versus the physician orders for lifesustaining treatment program. J Am Geriatr Soc. 2010;58(7): 1241-1248.

3. Department of Health and Human Services Office of Inspector General. Medicare Nursing Home Resident Hospitalization Rates Merit Additional Monitoring (Oei-06-11-00040). Nov 2013. Available at: https://oig.hhs.gov/ oei/reports/oei-06-11-00040.pdf. Accessed April 8, 2016.

4. Wenger NS, Citko J, O'Malley $\mathbf{K}$, et al. Implementation of physician orders for life sustaining treatment in nursing homes in California: evaluation of a novel statewide dissemination mechanism. Journal of General Internal Medicine. 2013;28(1):51-57.

5. Centers for Medicare \& Medicaid Services. Long Term Care Minimum Data Set (MDS). 2012. Available at: http://www.cms.gov/Research-StatisticsData-and-Systems/Files-for-Order/IdentifiableDataFiles/LongTermCareMinimumDataSetMDS.html. Accessed April 8, 2016.

6. California Emergency Medical Services Authority. Physician Orders for Life-Sustaining Treatment (POLST). 2011. Available at: http://www.cdph. ca.gov/programs/LnC/Documents/MDS30-ApprovedPOLSTForm.pdf. Accessed June 29, 2015

7. State of California-Health and Human Services Agency, California Department of Public Health MDS 3.0 California Section S April 2011. Available at: https://www.cdph.ca.gov/programs/LnC/Documents/MDS-CA-Section-S-4-1-2011.PDF. Accessed February 24, 2016.

8. Chodosh J, Edelen MO, Buchanan JL, et al. Nursing home assessment of cognitive impairment: development and testing of a brief instrument of mental status. J Am Geriatr Soc. 2008;56(11):2069-2075.

9. Morris JN, Fries BE, Morris SA. Scaling ADLS within the MDS. J Gerontol A Biol Sci Med Sci. 1999;54(11):M546-M553.

10. RTI International. MDS 3.0 Quality Measures User's Manual 2012. v5.0 0301-2012. Available at: https://www.cms.gov/Medicare/Quality-InitiativesPatient-Assessment-Instruments/NursingHomeQualityInits/downloads / MDS30QM-Manual.pdf. Accessed April 8, 2016.

11. Centers for Medicare \& Medicaid Services. MDS 3.0 RAI Manual, October 2013, Chapter 2, Assessments for the RAI. 2013. Available at: https:/ /www. cms.gov/Medicare/Quality-Initiatives-Patient-Assessment-Instruments/ NursingHomeQualityInits/MDS30RAIManual.html. Accessed April 8, 2016 .
12. California State. Assembly Bill No. 3000, Ch. 266. 2007-2008. Available at: http://www.leginfo.ca.gov/pub/07-08/bill/asm/ab_2951-3000/ab_ 3000_bill_20080804_chaptered.pdf. Accessed June 29, 2015.

13. State of California-Health and Human Services Agency, California Department of Public Health All Facilities Letter 10-42. 2010.

14. Stata Statistical Software: Release 13 [computer program]. College Station, TX: StataCorp LP2013.

15. Shepardson LB, Gordon HS, Ibrahim SA, Harper DL, Rosenthal GE. Racial variation in the use of do-not-resuscitate orders. Journal of General Internal Medicine. 1999;14(1):15-20.

16. Loggers ET, Maciejewski PK, Paulk E, et al. Racial differences in predictors of intensive end-of-life care in patients with advanced cancer. Journal of Clinical Oncology: Official Journal of the American Society of Clinical Oncology. 2009;27(33):5559-5564.

17. Quill CM, Ratcliffe SJ, Harhay MO, Halpern SD. Variation in decisions to forgo life-sustaining therapies in US ICUs. Chest. 2014;146(3):573-582.

18. Hickman SE, Nelson CA, Moss AH, Tolle SW, Perrin NA, Hammes BJ. The consistency between treatments provided to nursing facility residents and orders on the physician orders for life-sustaining treatment form. J Am Geriatr Soc. 2011;59(11):2091-2099.

19. Phillips RS, Wenger NS, Teno J, et al. Choices of seriously ill patients about cardiopulmonary resuscitation: correlates and outcomes. Support investigators. Study to understand prognoses and preferences for outcomes and risks of treatments. Am J Med. 1996;100(2):128-137.

20. Levin JR, Wenger NS, Ouslander JG, et al. Life-sustaining treatment decisions for nursing home residents: who discusses, who decides and what is decided? J Am Geriatr Soc. 1999;47(1):82-87.

21. Rosenfeld KE, Wenger NS, Kagawa-Singer M. End-of-life decision making: a qualitative study of elderly individuals. Journal of General Internal Medicine. 2000;15(9):620-625.

22. Patrick DL, Starks HE, Cain KC, Uhlmann RF, Pearlman RA. Measuring preferences for health states worse than death. Medical Decision Making: An International Journal of the Society for Medical Decision Making. 1994; 14(1):9-18.

23. Resnick HE, Foster GL, Hickman SE. Nursing home participation in endof-life programs: United States, 2004. The American Journal of Hospice \& Palliative Care. 2009;26(5):354-360.

24. Saliba D, Buchanan J. Development and Validation of a Revised Nursing Home Assessment Tool: MDS 3.0 Health. CMS MDS 3.0 Validation Contract No. 500-00-0027/Task Order \#2. April 2008. Available at: http://www.cms. gov/Medicare/Quality-Initiatives-Patient-Assessment-Instruments/NursingHomeQualityInits/downloads/MDS30FinalReport.pdf. Accessed April 8, 2016. 